\title{
APLICACIÓN DE ULTRASONIDOS PARA EL CONTROL DE ALGAS EN AGUAS REGENERADAS ALMACENADAS EN BALSAS DE RIEGO
}

\author{
Maestre Valero, J.F. ${ }^{1}$,Pedrero Salcedo, $F^{2}{ }^{2}$,Soto García, M. ${ }^{3}$, Alarcón J.J. ${ }^{4}$
}

\begin{abstract}
${ }^{1}$ Investigador, Departamento de Riego, Centro de Edafología y Biología Aplicada del Segura (CEBAS-CSIC), Campus Universitario de Espinardo, 30100, Murcia, España, Tel: +34968396223. Fax: +34-968396213, Email: imaestre@cebas.csic.es

2 Investigador, Departamento de Riego, Centro de Edafología y Biología Aplicada del Segura (CEBAS-CSIC), Campus Universitario de Espinardo, 30100, Murcia, España, Tel: +34968396223. Fax: +34-968396213, Email: fpedrero@cebas.csic.es

${ }^{3}$ Dirección técnica, Comunidad de Regantes del Campo de Cartagena. Paseo Alfonso XIII, 22, 30201 Cartagena, Murcia, Spain, Tel: +34-968514200, Email: mariano.soto@crcc.es

${ }^{4}$ Investigador, Departamento de Riego, Centro de Edafología y Biología Aplicada del Segura (CEBAS-CSIC), Campus Universitario de Espinardo, 30100, Murcia, España, Tel: +34968396303. Fax: +34-968396213, Email: jalarcon@cebas.csic.es
\end{abstract}

\section{Resumen}

En las regiones áridas y semiáridas las balsas de riego son infraestructuras que garantizan el suministro de agua a los cultivos. Sin embargo, favorecen la proliferación de algas provocando serios problemas en la calidad del agua que afectan negativamente a los sistemas de riego (filtración, emisores, elementos auxiliares,...). Ante esta problemática, el objetivo de este estudio fue evaluar, a escala real, el efecto de los ultrasonidos sobre parámetros físico-químicos y microbiológicos de interés agronómico en el agua de riego almacenada y sobre la presencia de algas en el agua.

El ensayo se realizó en tres balsas de aguas regeneradas y una de agua suministrada por la Comunidad de Regantes del Campo de Cartagena, principalmente procedente del Trasvase Tajo-Segura. Equipos de emisión de ultrasonidos se instalaron en 2 de las 4 balsas para tratar de reducir la presencia de algas. Quincenalmente, se determinó la calidad del agua mediante una sonda multiparamétrica y el análisis de muestras de agua en laboratorio.

Los resultados del experimento manifiestan que los ultrasonidos controlan el crecimiento de microalgas manteniendo la concentración de éstas en valores aceptables para evitar problemas de obturación en los sistemas de riego y mejorando por tanto la calidad del agua almacenada.

\section{1- Introducción, Objetivos}

En las regiones áridas y semiáridas, como el sureste de España, la disponibilidad de agua de riego en cantidad y calidad suficiente se ha convertido en un factor limitante de la actividad agrícola que ha potenciado la competitividad por los recursos hídricos (Soto García et al., 2014). En estas regiones, donde la escasez y la disponibilidad de agua varían estacionalmente, las balsas de riego son una instalación común para garantizar el suministro 
de agua a los cultivos pues permiten amortiguar el desfase temporal que se produce entre la disponibilidad de los recursos y su demanda. En el caso particular de la Cuenca del Segura, región donde se ha realizado este ensayo, existen más de 15.000 balsas de riego (MaestreValero et al., 2011).

A pesar de los beneficios expuestos y de que son requisito fundamental para la sostenibilidad del riego en esta cuenca deficitaria, la gran cantidad de nutrientes que contienen las aguas de riego almacenadas en balsas, junto con las condiciones climáticas adecuadas, provocan el crecimiento y desarrollo de algas de tamaño microscópico que pueden provocar problemas técnicos y de calidad del agua (Karico, 2000; Brainwood et al., 2004; Sperling et al., 2008). Además, cuando el agua almacenada se destina a riego localizado, se producen frecuentemente obturaciones de los emisores que afectan principalmente a la uniformidad de aplicación de agua de riego en parcela, lo cual conduce a variaciones en el crecimiento y reducciones en la productividad del cultivo (Adin et al., 1989; Adin y Sacks, 1991; Bucks y Nakayama, 1991). Este último inconveniente se agudiza más aún cuando existe una concentración disponible de nutrientes elevada para el desarrollo de algas como es frecuente en las aguas regeneradas.

En la bibliografía se pueden encontrar diferentes técnicas de mejora de la calidad del agua almacenada dirigidas a reducir principalmente la presencia de micro y macro algas, como son las técnicas biológicas de implementación de paja de cebada (Purcell et al., 2012) o introducción de bacterias de secreción de enzimas líticas (Peng et al., 2003) en el agua, las técnicas químicas de adición de alguicidas como el sulfato de cobre o el permanganato potásico y las técnicas físicas como el uso de coberturas de sombreo suspendidas (Martínez-Alvarez et al., 2009) o flotantes (Headley y Tanner, 2012) y los ultrasonidos (Heng et al., 2007).

Entre estas técnicas, este estudio evaluó el efecto de los ultrasonidos sobre la presencia de algas y sobre los parámetros físico-químicos y microbiológicos de interés agronómico del agua regenerada almacenada en balsas de riego.

\section{2.- Materiales y métodos}

\section{Las balsas de riego y los ultrasonidos}

El ensayo se llevó a cabo en cuatro balsas de riego. Tres de ellas almacenaron aguas regeneradas (San Javier; SJ, Alcázares 1; AC1 y Alcázares 2; AC2) y en dos de las balsas que almacenaron aguas regeneradas se instalaron equipos de ultrasonidos (1 equipo por balsa; modelo TISU XXI). Los equipos se instalaron a principios de junio. La balsa restante almacenó agua del transvase Tajo - Segura; TR. Los sistemas de tratamiento de ultrasonidos para el control de algas son sistemas submarinos de sonido, de alta especificidad y que emiten ondas de sonido ultrasónico para destruir las algas. Las ondas originan la rotura de los diferentes orgánulos celulares tales como las vacuolas, la pared celular o la membrana y las vesículas de gas de las algas verde-azuladas (Rajasekhar et al., 2012). La tabla 1 muestra las características de las balsas.

Tabla 1. Características de las balsas seleccionadas en el experimento.

\begin{tabular}{|c|c|c|c|c|}
\hline Balsa & Localización & Tipo de agua & $\begin{array}{c}\text { Dimensiones } \\
(\mathrm{L} \times \mathrm{A} \times \mathrm{P})\end{array}$ & $\begin{array}{c}\text { Caudal depuradora } \\
\left(\mathrm{m}^{3} / \mathrm{día}\right)\end{array}$ \\
\hline $\begin{array}{c}\text { La Trinchera } \\
\text { (TR) }\end{array}$ & $\begin{array}{c}37^{\circ} 48^{\prime} \mathrm{N} \\
0^{\circ} 55^{\prime} \mathrm{W}\end{array}$ & Tajo - Segura & $450 \times 120 \times 6$ & - \\
\hline $\begin{array}{c}\text { San Javier } \\
\text { (SJ) }\end{array}$ & $\begin{array}{c}37^{\circ} 47^{\prime} \mathrm{N} \\
0^{\circ} 49^{\prime} \mathrm{W}\end{array}$ & Regenerada & $120 \times 70 \times 4$ & $\begin{array}{c}\text { Invierno }=7.000 \\
\text { Verano }=15.000\end{array}$ \\
\hline $\begin{array}{c}\text { Alcázares } 1^{*} \\
\text { (AC1) }\end{array}$ & $\begin{array}{c}37^{\circ} 44^{\prime} \mathrm{N} \\
0^{\circ} 53^{\prime} \mathrm{W}\end{array}$ & Regenerada & $110 \times 105 \times 2$ & $\begin{array}{c}\text { Invierno }=5.000 \\
\text { Verano }=10.000\end{array}$ \\
\hline $\begin{array}{c}\text { Alcázares } 2^{*} \\
\text { (AC2) }\end{array}$ & $\begin{array}{c}37^{\circ} 44^{\prime} \mathrm{N} \\
\left.0^{\circ} 53^{\prime} \mathrm{W}\right)\end{array}$ & Regenerada & $100 \times 13 \times 2$ & $\begin{array}{c}\text { Invierno }=5.000 \\
\text { Verano }=10.000\end{array}$ \\
\hline
\end{tabular}

*Instalación de un equipo de ultrasonidos 


\section{Descripción del ensayo y calidad del agua}

El experimento se realizó durante 3 meses, desde final de mayo a final de agosto de 2014. Cada dos semanas se recogieron muestras de agua de cada balsa (1L), que se transportaron directamente al laboratorio en nevera portátil y se almacenaron a $5^{\circ} \mathrm{C}$ antes de ser procesadas. Se realizaron análisis en el laboratorio tanto físico-químico como microbiológico. Los análisis fisicoquímicos consistieron en la determinación mediante (ICPOES ICAP 6500 Duo Thermo, Inglaterra) de la concentración de los macronutrientes ( $\mathrm{Na}, \mathrm{K}$, $\mathrm{Ca}, \mathrm{Mg}$ ), micronutrientes (Fe, B, Mn) y metales pesados (Ni, Cd, Cr, Cu, Pb, $\mathrm{Zn}$ ) y los sólidos en suspensión (filtrado a vacío en un matraz Kitasato con embudo Buchner y pesada de filtros; poro $=0,47 \mathrm{~mm}$ y estufa a $105^{\circ} \mathrm{C}$ ). Los análisis microbiológicos consistieron en la detección del número total de $E$. coli presente en un volumen de $100 \mathrm{~mL}$, por el método de filtración por membrana. Además, se realizaron registros para el perfil de profundidad de la calidad del agua in situ mediante una sonda multiparamétrica modelo Hidrolab DS.5 (OTT hidromet, Kempten, Alemania) dotada de sensores para determinar temperatura, conductividad eléctrica (CE) y clorofila-a (Cl-a; proxy de microalgas). Las muestras y sondeos se tomaron y realizaron siempre en el espacio horario de entre la 13:00 h y 16:00 h.

Adicionalmente, se evaluó de forma visual la evolución de la cantidad de macrófitas en las balsas de riego durante el periodo de ensayo.

\section{3.- Resultados y discusión}

Los parámetros físico-químicos analizados in situ no presentaron variaciones en el perfil de profundidad, de modo que los resultados para estos parámetros muestran el valor medio para todo el perfil de profundidad en cada fecha de muestreo.

\section{Conductividad eléctrica (CE)}

Las balsas AC1, AC2 y SJ presentaron valores más elevados de salinidad que TR, durante el periodo de muestreo (Fig. 1a). La evolución de la CE en las tres balsas de almacenamiento de aguas regeneradas fue similar, mostrando una reducción progresiva en la época estival (debido al aumento del caudal tratado) y un aumento al finalizar la misma (Fig. 1b).
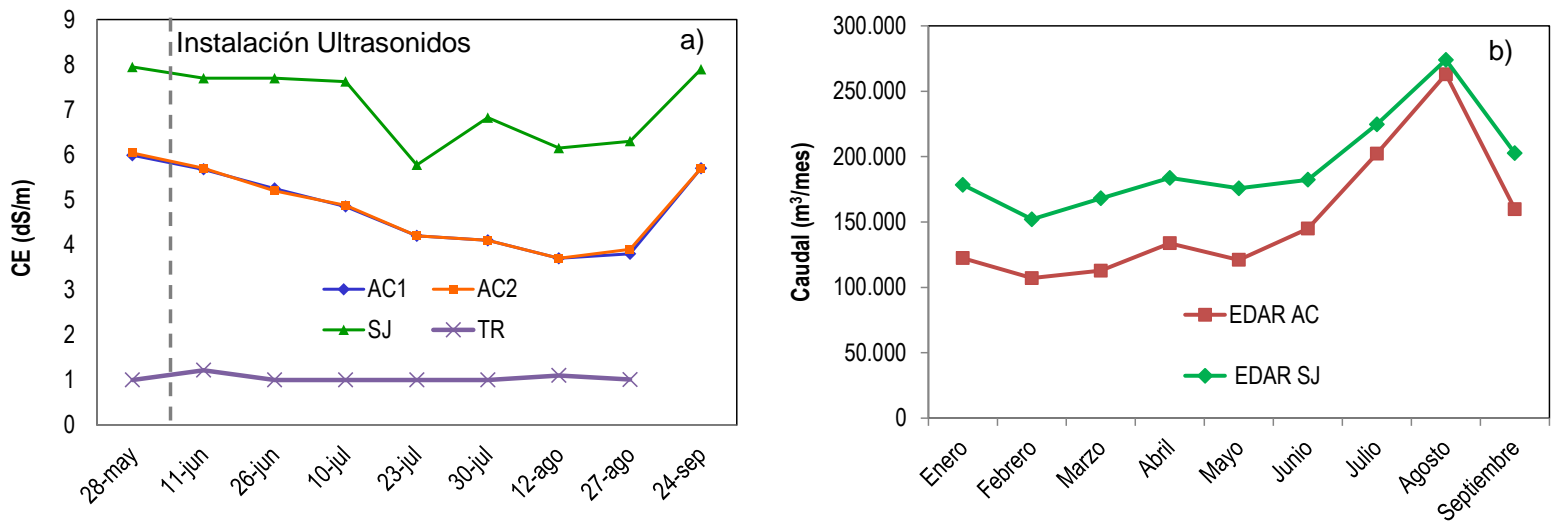

Figura 1. Evolución de (a) la conductividad eléctrica (CE) para las balsas $A C 1, A C 2, S J Y$ TR y (b) del caudal de las EDAR de Los Alcázares y San Javier en el periodo de muestreo. 
La concentración de $\mathrm{Cl}$-a (proxy de microalgas) en todos las balsas ensayadas permaneció en un rango de valores entre 5 - $15 \mu \mathrm{g} / \mathrm{l}$; excepto en AC1, AC2 y SJ al inicio del ensayo antes de la instalación de los equipos de ultrasonidos en AC1 y AC2. Durante los dos primeros muestreos (mayo y junio), la concentración de $\mathrm{Cl}-\mathrm{a}$ de la balsa SJ presentó valores elevados $(35-40 \mu \mathrm{g} / \mathrm{l})$ y la concentración de $\mathrm{Cl}-\mathrm{a}$ en las balsas AC1 y AC2 osciló entre 20 - $30 \mu \mathrm{g} / \mathrm{l}$ (Fig. 2a). Una vez se instalaron los equipos de ultrasonidos, se observó una reducción significativa en la concentración de $\mathrm{Cl}$-a y por tanto de microalgas en las balsas AC1 y AC2 que alcanzó a finales de junio valores en torno a $5 \mu \mathrm{g} / \mathrm{l}$. A pesar de que en la balsa SJ no se instaló ningún equipo de ultrasonidos, se detectó una reducción similar en la concentración de $\mathrm{Cl}$-a a las balsas AC1 y AC2. Esta reducción se atribuyó principalmente a las frecuentes limpiezas manuales de las algas (Potamogeton Pectinatus y Cladophora Glomerata) en la balsa SJ realizadas por los operarios de la EDAR (Figura 3).

Como era de esperar, la concentración de sólidos en suspensión (SS) mostró una cierta relación con la concentración de $\mathrm{Cl}$-a (Fig. 2b). Esta relación fue más elevada para las balsas que mostraron una mayor cantidad de macroalgas en descomposición (SJ > AC1 $>$ AC2 > TR). Es importante señalar que solamente en el segundo muestreo la balsa SJ presentó valores de SS superiores a los máximos establecidos por el Real Decreto 1620/2007 que regula la reutilización de las aguas regeneradas para usos agrícolas en el caso 2.1
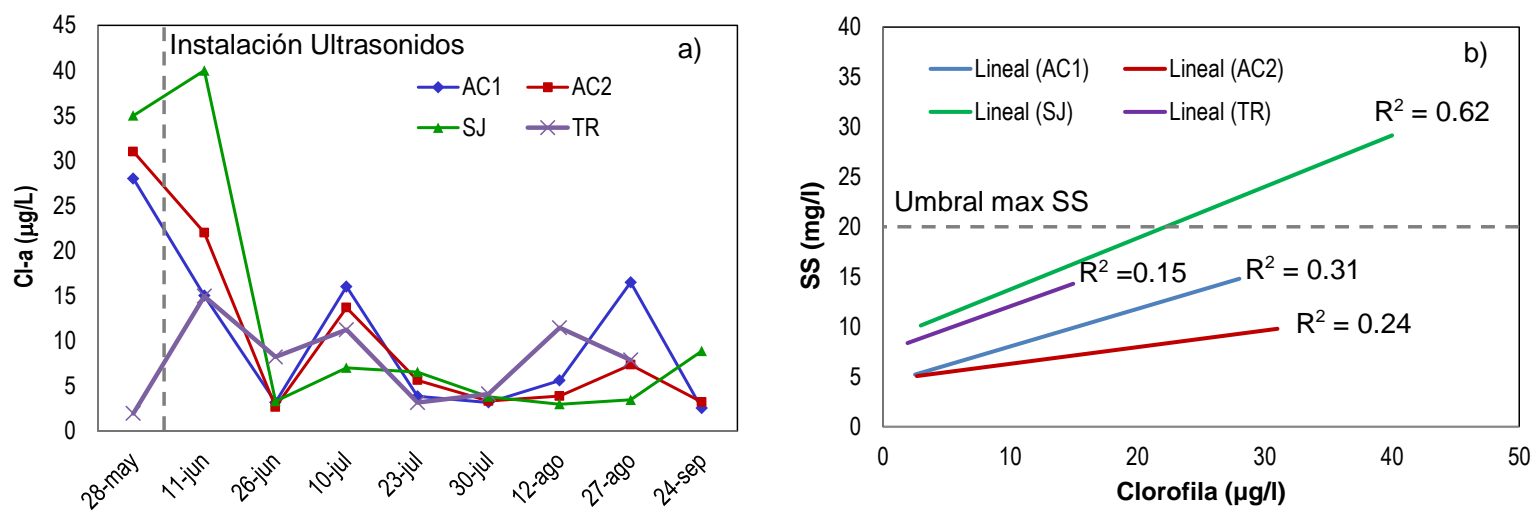

Figura 2. Evolución de (a) la concentración de la clorofila-a y (b) regresiones lineales entre la concentración de clorofila-a y los sólidos en suspensión para las balsas AC1, AC2, SJ Y

TR en el periodo de muestreo.
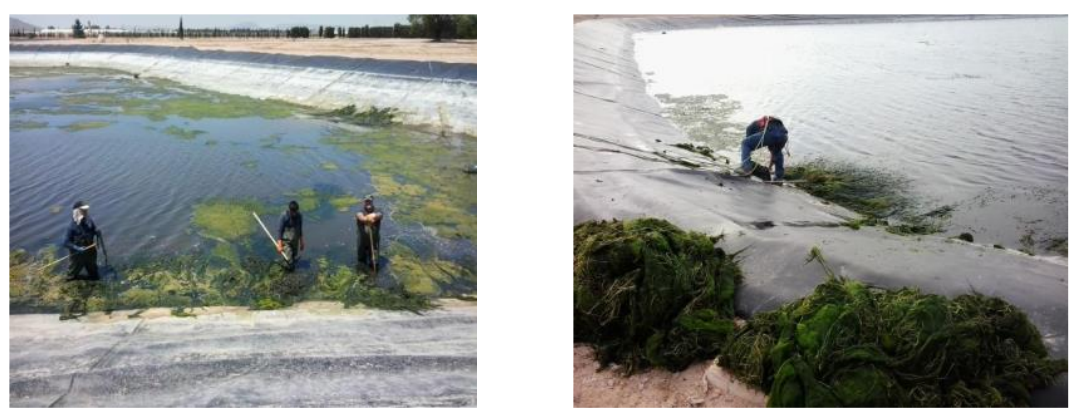

Figura 3. Limpieza manual de la balsa SJ durante el periodo del ensayo.

\section{Escherichia coli (E-coli)}


La concentración de E-coli se encontró bajo el valor máximo admisible del Real Decreto 1620/2007 para el uso agrícola más restrictivo (100 UFC/100 mL). El valor medio de la concentración de E-coli durante el periodo de ensayo y a partir de la instalación de los equipos de ultrasonidos fue de 17,4 UFC/100 mL y 23,4 UFC/100 mL en las balsas AC1 y AC2, respectivamente, mientras que en las balsas SJ y TR estos valores fueron algo superiores $32,5 \mathrm{UFC} / 100 \mathrm{~mL}$ y $31,9 \mathrm{UFC} / 100 \mathrm{~mL}$, respectivamente. Los valores inferiores de E-coli en las balsas AC1 y AC2 se atribuyen a un efecto combinado de la aplicación de ultrasonidos (Toscano et al., 2013) y a un proceso de depuración natural producido por la incidencia de la radiación solar (Sala y Millet, 1997).

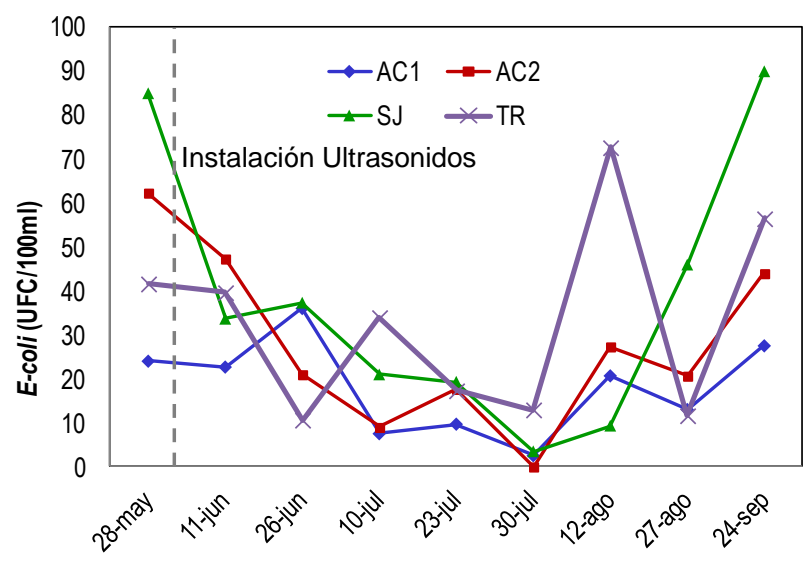

Figura 3. Evolución de la concentración E-coli para las balsas $A C 1, A C 2$, SJ Y TR en el periodo de muestreo.

\section{Elementos nutricionales}

Los macronutrientes como nitrógeno y fósforo son una preocupación ambiental por sus roles como factores limitantes en el crecimiento de plantas acuáticas que pueden causar la degradación de la calidad del agua y la aceleración del proceso de eutrofización. En las balsas de aguas regeneradas estudiados, la concentración de $\mathrm{PO}_{4}{ }^{3-}$ fue superior al límite establecido de riesgo de eutrofización (1,5 mg/l) (Sawyer et al., 1994), particularmente en SJ donde tanto las concentraciones de $\mathrm{NO}_{3}{ }^{-}, \mathrm{PO}_{4}{ }^{3-}$ y $\mathrm{K}$ fueron ligeramente superiores a los de AC1 y AC2 (Tabla 2). Tras comparar los valores de $\mathrm{NO}_{3}{ }^{-}, \mathrm{PO}_{4}{ }^{3-}$ y $\mathrm{K}$ del efluente proporcionados por ESAMUR (Entidad de Saneamiento y Depuración de Aguas Residuales de la Región de Murcia; datos no mostrados), no se observó ningún efecto del embalsamiento ni de los ultrasonidos en la concentración de los mismos, debido probablemente al corto tiempo de residencia de las aguas de riego en las balsas. En la balsa $T R$, los valores durante todo el ensayo fueron inferiores a los límites de riesgo de eutrofización.

En el caso de los elementos fitotóxicos, $\mathrm{Cl}^{-}$y $\mathrm{Na}^{+}$presentaron concentraciones muy superiores en las balsas con agua regenerada, especialmente la balsa SJ. En cuanto al B, se observó una concentración de $1 \mathrm{mg} / \mathrm{L}$ en las balsas $\mathrm{AC}$ y SJ. Estos valores elevados de $\mathrm{Na}, \mathrm{Cl}^{-}$y B podrían inducir toxicidad en cultivos como por ejemplo naranjo, melocotonero, alcachofa, etc. (Navarro, 2010).

Tabla 1. Valores medios de concentración de macronutrientes, micronutrientes y aniones en las balsas (TR, AC1, AC2 y SJ) durante el ensayo.

\begin{tabular}{|l|l|l|l|l|}
\hline & TR & AC1 & AC2 & SJ \\
\hline
\end{tabular}


XXXIII Congreso Nacional de Riegos

Universitat Politècnica de València, Valencia 2015

DOI:http://dx.doi.org/10.4995/CNRiegos.2015.1439

\begin{tabular}{|c|c|c|c|c|}
\hline & \multicolumn{4}{|c|}{ Macronutrientes } \\
\hline $\mathrm{Na} \mathrm{(mg/L)}$ & 66,51 & 729,18 & 753,52 & 1218,03 \\
\hline $\mathrm{K}(\mathrm{mg} / \mathrm{L})$ & 6,19 & 39,91 & 40,31 & 56,55 \\
\hline $\mathrm{Ca}(\mathrm{mg} / \mathrm{L})$ & 81,87 & 151,28 & 151,6 & 156,28 \\
\hline $\mathrm{Mg}(\mathrm{mg} / \mathrm{L})$ & 44,12 & 148,63 & 153,35 & 201,83 \\
\hline $\mathrm{P}(\mathrm{mg} / \mathrm{L})$ & 0,10 & 1,92 & 1,85 & 2,66 \\
\hline $\mathrm{S}(\mathrm{mg} / \mathrm{L})$ & 78,20 & 223,28 & 224,92 & 232,02 \\
\hline & \multicolumn{5}{|c|}{ Micronutrientes } \\
\hline $\mathrm{B}(\mathrm{mg} / \mathrm{L})$ & 0,11 & 0,92 & 0,91 & 0,88 \\
\hline $\mathrm{Mn}(\mathrm{mg} / \mathrm{L})$ & 0,23 & 0,61 & 0,61 & 0,77 \\
\hline & \multicolumn{5}{|c|}{ Aniones } \\
\hline $\mathrm{Cl}^{-}(\mathrm{mg} / \mathrm{L})$ & 99,1 & 1132,8 & 1054,8 & 1899,4 \\
\hline $\mathrm{NO}_{3}{ }^{-}(\mathrm{mg} / \mathrm{L})$ & 0,93 & 2,32 & 2,34 & 4,72 \\
\hline $\mathrm{PO}^{3-}(\mathrm{mg} / \mathrm{L})$ & 1,00 & 5,41 & 4,78 & 6,28 \\
\hline $\mathrm{SO}_{4}{ }^{2-}(\mathrm{mg} / \mathrm{L})$ & 233,9 & 677,8 & 628,9 & 671,9 \\
\hline
\end{tabular}

\section{Comparativa económica ultrasonidos vs limpieza manual}

La balsa SJ requirió limpieza manual de macroalgas durante los meses en los que las temperaturas son más altas en la zona, época en la que el crecimiento de algas supone un problema, pese a la mayor tasa de renovación del agua. Las limpiezas se sucedieron durante 5 meses aproximadamente con una periodicidad de 4 semanas. Se ha identificado que fueron necesarias 5 limpiezas durante el periodo de tiempo del ensayo, además del mes anterior y el mes siguiente. Cada limpieza tuvo una duración de 5 días, y participaron 4 operarios, que dedicaron su jornada laboral al completo $(7 \mathrm{~h})$ a la limpieza de la balsa. El salario de un operario se ha fijado en $15 € / \mathrm{h}$. Por lo tanto, se estima el coste de cada limpieza en $2.100 €$ ( 5 días $\cdot 4$ operarios $\cdot 7 \mathrm{~h} /$ operario $\cdot 15 € / \mathrm{h}$ ). Por consiguiente, el monto económico de las 5 limpiezas realizadas asciende a $10.500 €$. Este gasto se considera anual, ya que cada año se deberán realizar estas limpiezas de macroalgas coincidiendo con el incremento de las temperaturas del agua.

Por otro lado, las balsas AC1 y AC2 usaron los equipos de ultrasonidos para el control del crecimiento de algas en ellos. El coste de inversión para la instalación completa del equipo de ultrasonidos fue de $10.000 €$, (4.500 $€$ coste de los dos equipos de ultrasonidos y $5.500 €$ el de la obra civil en su caso e instalación eléctrica necesaria). El consumo energético total de los dos aparatos de ultrasonidos es de $300 \mathrm{Wh}$ y el precio medio del kWh se ha fijado en $0,12 €$, por lo que el consumo energético por hora de los aparatos de ultrasonidos es de 0,036 €/h. Al año, el coste de la energía consumida por los aparatos ascendería a $315 €$ aproximadamente. Además, el mantenimiento anual eléctrico y de limpieza que pueden requerir estos aparatos de ultrasonidos se estima en $1 \mathrm{~h}$ de trabajo de un técnico cada 15 días aproximadamente, lo que supondría un coste de $360 €$.

Al año, entre consumo energético y mantenimiento, los aparatos de ultrasonidos de las

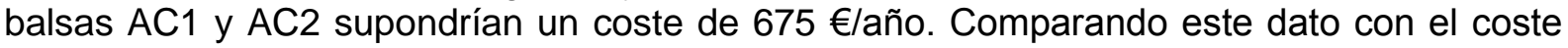
de limpieza manual de la balsa de SJ (10.500 €/año), se puede afirmar que en un año aproximadamente la inversión que supone la implantación del aparato de ultrasonidos en la balsa de SJ quedaría totalmente amortizada. La tabla 3 muestra la comparativa económica de ambos métodos; ultrasonidos y limpieza manual. 
Tabla 3. Datos económicos de la limpieza manual de la balsa SJ y de los equipos de ultrasonidos de AC1 y AC2.

\begin{tabular}{|c|c|c|c|}
\hline \multicolumn{2}{|c|}{ Limpieza manual } & \multicolumn{2}{|c|}{ Equipo de Ultrasonidos } \\
\hline Coste & & \multicolumn{2}{|c|}{ Inversión inicial } \\
\hline \multicolumn{2}{|c|}{ Coste por cada limpieza } & Equipos & $4.500 €$ \\
\hline 4 operarios & \multirow{4}{*}{$2.100 €$} & Obra e instalación eléctrica & $5.500 €$ \\
\hline 5 días & & Total Inversión & $10.000 €$ \\
\hline 7 h/día & & Coste energético & \\
\hline $15 € / h$ & & Consumo (300 Wh) & \\
\hline 5 limpiez & & Coste $\mathrm{kW} \cdot \mathrm{h}(0,12 €)$ & $315 €$ \\
\hline TOTAL L. MANUAL & $10.500 €$ & Activo 365 días $24 \mathrm{~h}$ & \\
\hline & & Coste limpieza equip & nual) \\
\hline & & № Limpiezas/año (24) & \\
\hline & & Tiempo limpieza (1h) & $360 €$ \\
\hline & & Coste hora $(15 €)$ & \\
\hline & & TOTAL ULTRASONIDOS & $10.675 €$ \\
\hline
\end{tabular}

\section{4.- Conclusiones y recomendaciones}

Aunque el almacenamiento de las aguas de riego produce múltiples beneficios tanto hidráulicos (regulación del agua disponible) como físico-químicos (reducción de sólidos en suspensión, etc.), como biológicos (desinfección solar), en regiones semi-áridas con un alto potencial agrícola como la Región de Murcia, el crecimiento de algas puede suponer un factor determinante en el deterioro de la calidad del agua de riego.

La implantación de equipos de ultrasonidos en balsas de riego permite controlar el crecimiento de microalgas, manteniendo la concentración de éstas en valores aceptables para evitar problemas de obturación en los sistemas de riego. La concentración de sólidos en suspensión presentes en las balsas estudiadas presentó cierta relación con la concentración de clorofila-a (microalgas). A nivel de macroalgas, la aplicación de ultrasonidos mejoró la calidad del agua almacenada, aunque no es capaz por sí solo de erradicarlas completamente.

No se ha observado ningún efecto de los ultrasonidos sobre la concentración de macronutrientes, micronutrientes, aniones y la conductividad eléctrica.

La combinación de ultrasonidos junto con la probable desinfección natural del agua por efecto de la radiación solar ha mostrado ser eficaz para evitar el crecimiento de E-coli en las balsas de riego por encima de los umbrales legalmente permitidos por decreto.

El coste económico que supone la implantación de un equipo de ultrasonidos, se podría amortizar en un año aproximadamente, comparando con el coste que supone la extracción manual de algas en las balsas.

\section{5.- Bibliografía}


Adin, A. \& Sacks, M. (1991). Dripper clogging factors in wastewater irrigation. Journal of Irrigation and Drainage Engineering. 117 (6), 813-826.

Brainwood, A., Burgin, S. \& Maheshwari, B. (2004). Temporal variations in water quality of farm dams: impacts of land use and water sources. Agricultural Water Management. 70, 151-175.

Bucks, D.\& Nakayama, F. (1991). Water quality in drip/trickle irrigation: A review. Irrigation Science. 12 (4), 187-192.

Heng, L., Jun, N., Wen-jieb H. \& Guibaia, L. (2007). Algae removal by ultrasonic irradiation-coagulation. Desalination 239,191-197.

Karico (2000). Technical report (I): the survey of pollution sources of water for the agricultural use (in Korean). Korean Agricultural and Rural Infrastructure Cooperation, pp. 1626.

Maestre Valero, José Francisco (2011). Efectos de la aplicación de coberturas de sombreo suspendidas sobre balsas de riego. Tesis doctoral. Universidad Politécnica de Cartagena.

Navarro, T.M. (2010). Reutilización de aguas regeneradas. Aspectos tecnológicos y jurídicos. Fundación Instituto Euromediterráneo del Agua.

Purcell, D., Parsons, S.A., Jefferson, B., Holden, S., Campbell, A., Wallen, A., Chipps, M., Holden, B. \& Ellingham, A. (2012). Experiences of algal bloom control using green solutions barley straw and ultrasound, an industry perspective. Water and Environment Journal 2, 148-56.

Peng, C., Wu, G., Xi, Y., Xia, Y., Zhang, T. \& Zhao, Y. (2003). Isolation and identification of three algae-lysing bacteria and their lytic effects on bluegreen algae (cyanobacteria). Research of Environmental Sciences 16, 37-40.

Rajasekhar, P., Fan, L., Nguyen, T. \& Roddick, F.A. (2012). A review of the use of sonication to control cyanobacterial Blooms. water research 46, 4319-4329

Sala, L. \& Millet, X. (1997). Aspectos básicos de la reutilización de las aguas residuales regeneradas para el riego de campos de golf. Apuntes de las jornadas técnicas del golf, 1995.

Sperling, E., Da Silva, A.C. \& Lodolf, L.N. (2008). Comparative eutrophication development in two Brazilian water supply reservoirs with respect to nutrient concentrations and bacteria growth. Desalination. 226, 169-174.

Sawyer, C., McCarty, P. L., \& Parkin, G.F. (1994). Chemistry for Environmental Engineering. Fourth Ed. p. 552-566 y 596-601.

Soto García, M., Martínez Álvarez, V. y Martín Górriz, B. (2014). El regadío en la Región de Murcia. Caracterización y análisis mediante indicadores de gestión. Sindicato Central de Regantes del Acueducto Tajo-Segura 268 pp.

Toscano, A., Hellio, C., Marzo, A., Milani, M., Lebret, K., Giuseppe, L., Cirelli, G.L.\& Langergraber, G. (2013). Removal efficiency of a constructed wetland combined with ultrasound and UV devices for wastewater reuse in agriculture. Environmental Technology, $34: 15,2327-2336$ 